\title{
Analysis of Patient-Perceived Nasal Appearance Evaluations Following Functional Septorhinoplasty With Spreader Graft Placement
}

Jennifer C. Fuller, MD; Patricia A. Levesque, BS; Robin W. Lindsay, MD

IMPORTANCE Spreader grafts have been shown to improve nasal airway obstruction in patients with nasal valve dysfunction; however, their use has been limited by concerns over their aesthetic penalty of widening the nasal dorsum.

OBJECTIVE To evaluate the association of functional septorhinoplasty (FSRP) using spreader graft placement with patient satisfaction with nasal appearance postoperatively.

DESIGN, SETTING, AND PARTICIPANTS This prospective cohort study was conducted in a university-based tertiary care medical center. All participants were patients undergoing FSRP for the treatment of nasal obstruction who had spreader grafts placed between June 2016 and May 2018.

EXPOSURES The Nasal Obstruction Symptom Evaluation (NOSE) scale and the FACE-Q Satisfaction With Nose, FACE-Q Satisfaction With Nostrils, and FACE-Q Social Functioning scales were administered to patients preoperatively and at 2, 4, 6, and 12 months postoperatively. Patient demographics, nasal history, and outcomes were analyzed.

MAIN OUTCOMES AND MEASURES Comparison of preoperative and postoperative NOSE and FACE-Q scores.

RESULTS A total of 154 patients (72 male [46.8\%]) with mean (SD) age of 36.8 (15.4) years underwent FSRP with spreader graft placement. Mean (SD) last follow-up was 5.8 (4.1) months postoperatively (range, 2-12 months). Fifty-seven patients had 6 months follow-up, and 42 patients had at least 12 months of follow-up. At time of last follow-up, mean (SD) NOSE and FACE-Q Satisfaction With Nose, Satisfaction With Nostrils, and Social Functioning scores significantly improved from 62.7 (20.7) to 22.8 (21.0) $(P<.001), 54.7$ (22.2) to 76.2 $(25.1)(P<.001), 59.4(27.9)$ to $83.6(24.8)(P<.001)$, and $73.6(21.8)$ to $81.7(21.9)(P<.001)$, respectively. When separated into those with only spreader grafts $(n=89)$ and those with spreader grafts plus other graft types $(n=65)$, there was no significant difference between score improvements in the 2 groups. There were no significant differences in final nasal appearance scores between patients undergoing functional vs dual functional and cosmetic FSRP.

CONCLUSIONS AND RELEVANCE Despite concerns that placement of spreader grafts for the treatment of nasal obstruction due to nasal valve dysfunction during FSRP may have a negative impact on nasal aesthetics, this study shows that both nasal obstruction and patient satisfaction with their nasal appearance were significantly improved following surgery.

LEVEL OF EVIDENCE 3.

Author Affiliations: Department of Otolaryngology, Harvard Medical School, Boston, Massachusetts (Fuller, Levesque, Lindsay); Department of Otolaryngology, Massachusetts Eye and Ear Infirmary, Boston (Fuller, Levesque, Lindsay): Department of Otolaryngology, University of Minnesota, Minneapolis (Fuller).

Corresponding Author: Jennifer C. Fuller, MD, University of Minnesota, 7373 France Ave, Ste 410, Minneapolis, MN 55435 (fulle551@umn.edu). 
$\mathrm{N}$ asal airway obstruction (NAO) negatively affects quality of life and is a leading complaint among patients presenting to otolaryngologists. ${ }^{1}$ Structural obstruction of nasal airflow is often caused by septal deviation, turbinate hypertrophy, and/or nasal valve dysfunction (NVD). The importance of identifying and treating NVD was highlighted in the American Academy of Otolaryngology-Head and Neck Surgery 2010 Clinical Consensus Statement, which described NVD as a distinct cause of NAO that can be surgically treated. ${ }^{2}$

Functional septorhinoplasty (FSRP) is frequently performed to straighten the septum and correct NVD. The types of grafts placed during surgery depend on the patient's specific anatomy, surgeon preference, and graft availability. Spreader grafts are a common type of graft used to treat internal nasal valve narrowing and have been shown to improve NAO symptoms. ${ }^{3,4}$ Nevertheless, both form and function must be considered, and spreader grafts are often criticized for causing undesirable widening of the nasal dorsum. However, to our knowledge, assessment of patients' satisfaction with their nasal appearance following spreader graft placement using a validated outcomes tool has not been performed.

The FACE-Q scale is a recently introduced, validated, multimodular patient-reported outcome instrument that measures a patient's perception of their nasal appearance and its impact on social functioning. ${ }^{5}$ In the present study, we seek to evaluate the association of FSRP using spreader graft placement with both NAO and patient satisfaction with their nasal appearance.

\section{Methods}

This study was performed at a single tertiary care medical center between June 2016 and May 2018 with institutional review board approval from the Human Subjects Research Committee of the Massachusetts Eye and Ear Infirmary. After we obtained written informed consent, patients presenting to the Massachusetts Eye and Ear Infirmary facial plastic surgery clinic were administered the Nasal Obstruction Symptom Evaluation (NOSE) survey and the FACE-Q Satisfaction With Nose, Satisfaction With Nostrils, and Social Functioning surveys preoperatively and at 2, 4, 6, and 12 months postoperatively. Surveys were administered in a manner compliant with the Health Insurance Portability and Accountability Act either in paper or electronic format at their scheduled clinic appointment or electronically via email through REDcap (Research Electronic Data Capture), an electronic data-capture platform designed for academic clinical and translational database development. ${ }^{6}$ All patients who underwent open FSRP with spreader graft placement by the senior author (R.W.L.) for the treatment of NAO and who completed both the NOSE and FACE-Q surveys both preoperatively and at 1 or more postoperative time points were included in this study. All patient demographics, nasal history, and outcomes were reported and analyzed.

A full description of the surgical technique used for each location of obstruction has been previously published. ${ }^{7}$ For spreader grafts in particular, a 2- to 3-mm-thick strip of either

\section{Key Points}

Question Is spreader graft placement for the treatment of nasal valve dysfunction associated with improved patient-perceived nasal appearance?

Findings This cohort study of 154 patients undergoing functional septorhinoplasty with spreader graft placement found that NOSE (Nasal Obstruction Symptom Evaluation), FACE-Q Satisfaction With Nose, and FACE-Q Satisfaction With Nostrils scores were improved significantly after surgery.

Meaning Despite concerns that spreader grafts have a negative aesthetic effect, the significant postoperative cosmetic improvements reported by the patients in this study after spreader graft septorhinoplasty suggest that this might be a viable technique for treatment of nasal valve dysfunction.

septal or costal cartilage is placed between the septum and the upper lateral cartilages. If a dorsal deviation is present, the upper lateral cartilages are released from the dorsum of the septum. The spreader grafts are then sutured to the dorsum of the septum using a 5-0 polydioxanone horizontal mattress suture. The upper lateral cartilages are then secured to the spreader grafts and the septum with an interrupted 5-0 polydioxanone suture to ensure that the caudal edge of the upper lateral cartilage has been fully extended to its normal anatomical length. The upper lateral cartilages are then sutured to the spreaders and the septum with horizontal mattress and interrupted sutures ensuring to secure the upper lateral cartilages slightly superior to the spreaders reconstituting the normal contour of the middle vault. If a dorsal deviation does not exist, often the upper lateral cartilages are not released, and the spreader grafts are placed in pockets between the upper lateral cartilages and the dorsum of the septum.

Perception of nasal appearance was measured using the FACE-Q Satisfaction With Nose, Satisfaction With Nostrils, and Social Functioning scales, which consist of 10, 5, and 8 validated questions, respectively, that the patient rates on a 4 -point Likert scale. Rasch transformation is used to transform the results into a score from 0 to 100, with higher scores indicating greater satisfaction with appearance or quality of life. ${ }^{5}$

The subjective symptoms of nasal obstruction were measured using the validated disease-specific quality-of-life outcomes measure, the NOSE questionnaire. This measure contains 5 questions related to nasal obstruction along a 5-point Likert scale, and responses are then converted to a total score from 0 (no nasal obstruction) to 100 (severe nasal obstruction). ${ }^{8}$

Statistical analyses were conducted using Microsoft Excel, version 15.28, and STATA 12.0. For all tests, $P<.05$ was considered significant. The minimal clinically important difference (MCID) for NOSE scores was set at 30 points, as previously described. ${ }^{9,10}$ While the MCIDs for the FACE-Q scores have not been established in the literature, this value was approximated using one-half of the mean baseline standard deviation (SD) as has been previously described. ${ }^{3,11}$ A 1-way analysis of variance with post hoc Tukey test was performed to determine statistically significant differences between preoperative and postoperative mean FACE-Q and NOSE scores at 


\begin{tabular}{|c|c|c|c|c|}
\hline \multirow[b]{2}{*}{ Survey Instrument } & \multicolumn{2}{|l|}{ Mean (SD) Score } & \multirow{2}{*}{$\begin{array}{l}\text { Mean (SD) } \\
\text { Change in Score }\end{array}$} & \multirow{2}{*}{$\begin{array}{l}\text { Clinically } \\
\text { Significant? }\end{array}$} \\
\hline & Preoperative (SD) & Postoperative (SD) & & \\
\hline \multicolumn{5}{|l|}{ NOSE } \\
\hline All patients & $62.7(20.7)$ & $22.8(21.0)$ & $-40.2(25.7)^{c}$ & Yes \\
\hline Spreader only & $62.3(18.7)$ & $22.1(21.1)$ & $-40.2(23.0)^{c}$ & Yes \\
\hline Spreader plus other & $63.4(23.4)$ & $23.8(21.0)$ & $-40.3(29.2)^{c}$ & Yes \\
\hline$P$ value $^{\mathrm{d}}$ & .77 & .64 & .97 & \\
\hline \multicolumn{5}{|c|}{ FACE-Q Satisfaction With Nose } \\
\hline All patients & $54.7(22.2)$ & $76.2(22.1)$ & $21.6(23.8)^{c}$ & Yes \\
\hline Spreader only & $51.8(20.0)$ & $72.8(23.3)$ & $21.5(25.9)^{\mathrm{c}}$ & Yes \\
\hline Spreader plus other & $58.2(24.7)$ & $79.0(20.5)$ & $21.7(20.9)^{c}$ & Yes \\
\hline$P$ value $^{\mathrm{d}}$ & .08 & .09 & .96 & \\
\hline \multicolumn{5}{|c|}{ FACE-Q Satisfaction With Nostrils } \\
\hline All patients & $59.4(27.9)$ & $83.6(23.1)$ & $24.8(27.7)^{c}$ & Yes \\
\hline Spreader only & $59.1(28.2)$ & $82.1(23.4)$ & $24.0(29.6)^{c}$ & Yes \\
\hline Spreader plus other & $59.9(27.9)$ & $85.6(22.7)$ & $25.7(25.2)^{c}$ & Yes \\
\hline$P$ value $^{\mathrm{d}}$ & .86 & .37 & .71 & \\
\hline \multicolumn{5}{|c|}{ FACE-Q Social Functioning } \\
\hline All patients & $73.6(21.8)$ & 81.7 (21.9) & $8.1(20.3)^{c}$ & No \\
\hline Spreader only & $72.5(22.0)$ & $80.9(23.3)$ & $8.5(22.1)^{c}$ & No \\
\hline Spreader plus other & $75.1(21.5)$ & $82.6(19.9)$ & $7.5(17.8)^{\mathrm{c}}$ & No \\
\hline$P$ value $^{\mathrm{d}}$ & .46 & .64 & .77 & \\
\hline
\end{tabular}

\author{
Abbreviations: MCID, mean clinically \\ important difference; NOSE, Nasal \\ Obstruction Symptom Evaluation. \\ a Reported are the mean (SD) \\ preoperative and postoperative \\ NOSE and FACE-Q scores at the \\ mean (SD) time of last follow-up of \\ 5.8 (4.1) months, by those with \\ spreader grafts only $(n=89)$, those \\ with spreader grafts plus other graft \\ types ( $n=65)$, and all patients \\ $(n=154)$. \\ ${ }^{\mathrm{b}}$ Clinical significance was determined \\ by a change exceeding the MCID for \\ each scale: NOSE score MCID, 30; \\ FACE-Q Satisfaction With Nose \\ MCID, 11.1; Satisfaction with Nostrils \\ MCID, 14.0; and Social Functioning \\ MCID, 10.9. \\ c Statistically significant change \\ within each cohort. \\ ${ }^{\mathrm{d}}$ The $P$ value rows indicate the \\ statistical difference between the 2 \\ spreader graft cohorts.
}

the various time points. Preoperative and postoperative NOSE and FACE-Q scores were compared using paired and unpaired $t$ tests as appropriate. Scores at the time of each patient's last follow-up were used to calculate means, unless otherwise specified.

\section{Results}

A total of 154 patients (72 male [46.8\%]) with a mean (SD) age of 36.8 (15.4) years underwent FSRP with spreader graft placement for the treatment of NAO. Of these patients, 87 (56.5\%) had a history of nasal fracture, and 60 (39.0\%) had prior nasal surgery. Of those with prior nasal surgery, 36 (60.0\%) had a prior septoplasty, 14 (23.3\%) had a prior septorhinoplasty, and $10(16.7 \%)$ had turbinoplasty. Mean (SD) time of last follow-up was 5.8 (4.1) months (range, 2-12 months). There were 57 patients with 6 months of follow-up and 42 patients with follow-up at 1 year.

The MCID was used to determine clinically significant changes, and for NOSE scores this was set at 30, as previously established. ${ }^{9,10}$ The FACE-Q MCID for Satisfaction With Nose, Satisfaction With Nostrils, and Social Functioning scores were estimated to be 11.1, 14.0, and 10.9, respectively, by distributional assessment, as has been previously described. ${ }^{3,11}$

Mean (SD) NOSE scores decreased by 40.2 (25.7) points at time of last follow-up, a clinically and statistically significant improvement (all supporting data reported in Table 1). FACE-Q Satisfaction With Nose, Satisfaction With Nostrils, and Social Functioning scores also had statistically significantly improvements at time of last follow-up, with a mean
(SD) change of 21.6 (23.8), 24.8 (27.7), and 8.1 (20.3) points, respectively $(P<.001$ for all; Table 1$)$. The change in FACE-Q Satisfaction With Nose and Satisfaction With Nostrils scores were clinically significant, while the change in Social Functioning was not (Table 1).

NOSE and FACE-Q scores at each follow-up time point are shown in Figure 1. For NOSE scores, FACE-Q Satisfaction With Nose, and FACE-Q Satisfaction With Nostrils, all postoperative measurements showed a significant improvement from baseline, and there was no significant difference between the postoperative time points, as supported by the data reported in Figure 1. Also shown in Figure 1 is a statistically significant improvement in mean FACE-Q Social Functioning scores from baseline at 2 and 4 months postoperatively, with clinically significant improvement at 4 months, but scores returned to baseline by 6 and 12 months, with no significant difference from baseline at these times.

Patients were then grouped into cohorts by those who received only spreader grafts $(n=89)$ and those who had other graft types in addition to spreader grafts $(n=65)$. Of the group receiving multiple graft types, 18 (28\%) had alar rim grafts, 24 (37\%) had lateral crural strut grafts, and 24 (37\%) had columellar strut grafts placed. There was no significant difference in scores or score improvements between the 2 groups (Table 1). As supported by the data reported in Table 1, both cohorts demonstrated statistically significant changes in all measures and clinically significant changes in all but FACE-Q social functioning.

Patients were also divided into cohorts by those who underwent purely functional procedures (FSRP) $(n=120)$ and those who had dual functional and cosmetic septorhinoplasty $(\mathrm{DFC})(\mathrm{n}=34)$. Examples of preoperative and postoperative 


\section{Figure 1. Preoperative and Postoperative NOSE and FACE-Q Scores at Each Measurement Point}

\section{A NOSE scores}

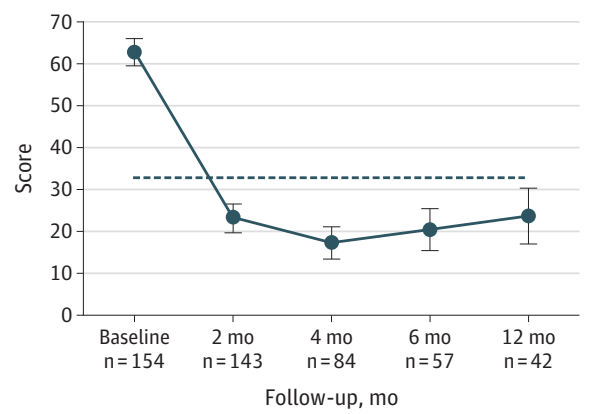

C FACE-Q Satisfaction With Nostrils

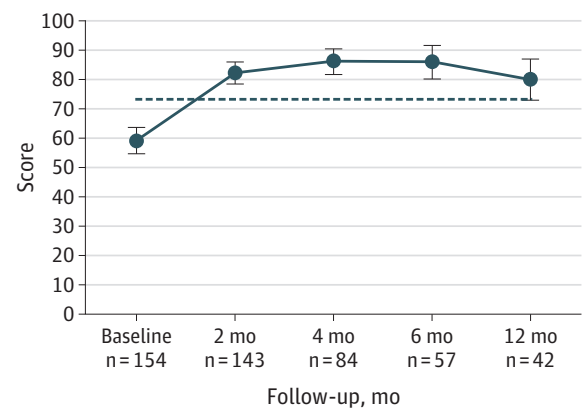

B FACE-Q Satisfaction With Nose

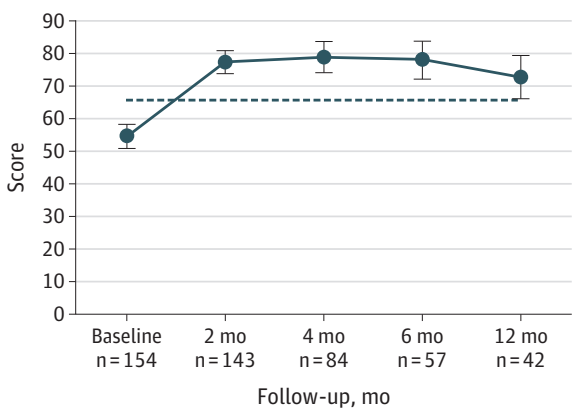

D FACE-Q Social Functioning

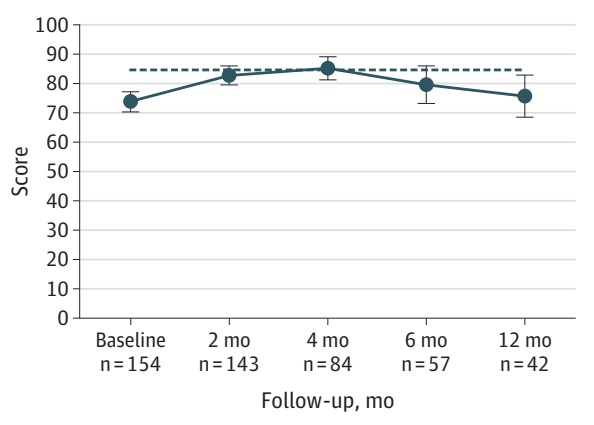

Baseline and 2-, 4-, 6-, and 12-month follow-up scores of Nasal Obstruction Symptom Evaluation (NOSE) scores (A) and FACE-Q scores (B-D). There are no significant differences between follow-up time point scores within each scale. All postoperative NOSE and FACE-Q Satisfaction With Nose and Satisfaction With Nostrils scores show clinically and statistically significant improvements from baseline. The FACE-Q Social Functioning score showed a statistically significant improvement at 2 and 4 months and clinically significant improvement at 4 months, but returned to baseline by

12 months.

Figure 2. Preoperative and Postoperative Nasal Photographs After Spreader Graft Placement Using Different Techniques

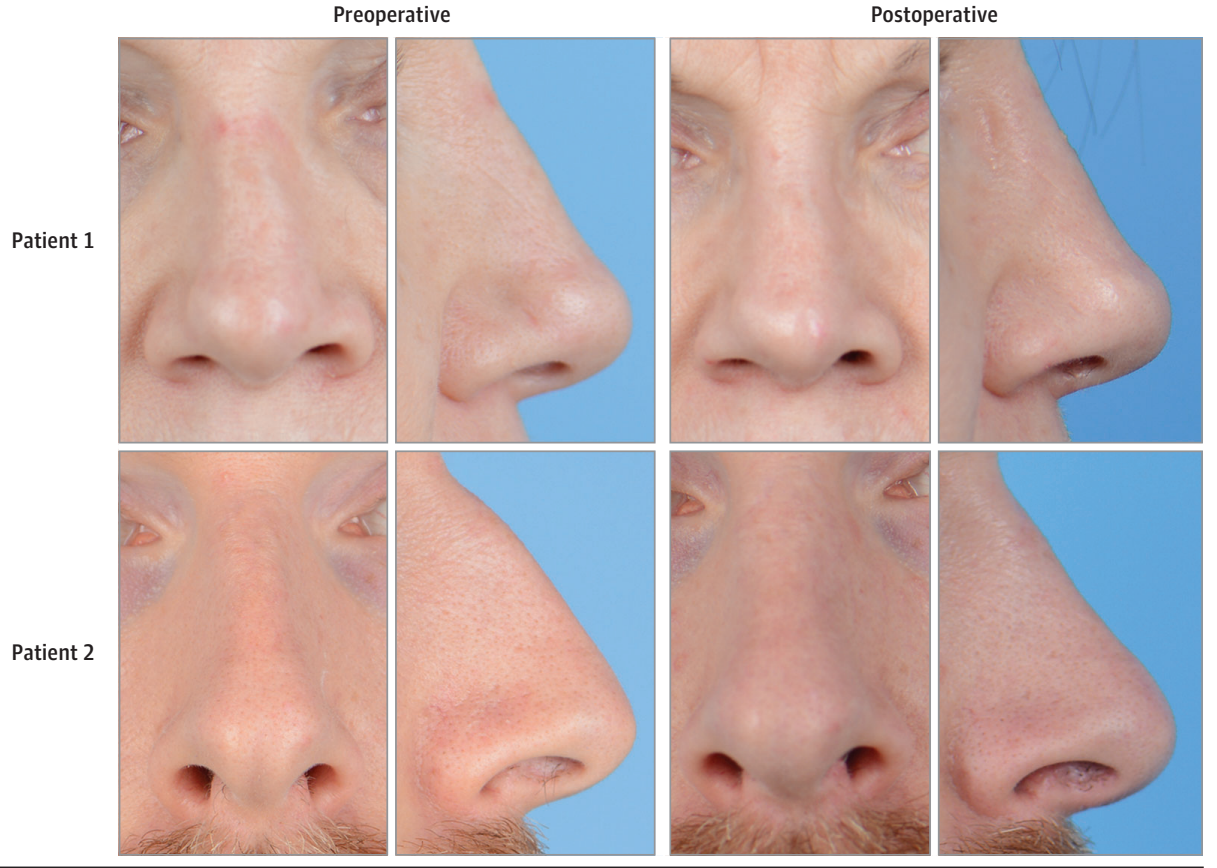

Patient 1 had a history of nasal trauma with depressed right middle vault and underwent open functional septorhinoplasty with placement of spreader grafts with release of the upper lateral cartilages from the septum to correct the narrow middle vault. Patient 2 had narrow internal nasal valves and a dorsal hump and underwent dual open functional and cosmetic septorhinoplasty with dorsal hump reduction and placement of spreader grafts. photographs after spreader graft placement using different techniques are shown in Figure 2. NOSE and FACE-Q scores at baseline and time of last follow-up are reported in Table 2 . There were no differences in NOSE scores between the cohorts, and both groups demonstrated statistically and clinically significant changes. As supported by the data reported in Table 2, patients undergoing DFC had significantly lower baseline FACE-QSatisfaction With Nose scores and significantly greater 


\begin{tabular}{|c|c|c|c|}
\hline \multirow[b]{2}{*}{ Rhinoplasty Type } & \multicolumn{2}{|c|}{ Score, Mean (SD) ${ }^{\mathrm{a}}$} & \multirow{2}{*}{$\begin{array}{l}\text { Score Change, } \\
\text { Mean (SD) }\end{array}$} \\
\hline & Baseline & Postoperative & \\
\hline \multicolumn{4}{|l|}{ NOSE } \\
\hline Functional & $62.8(20.9)$ & $21.8(20.3)$ & $-41.4(24.6)^{\mathrm{b}, \mathrm{c}}$ \\
\hline Dual functional + cosmetic & $62.5(20.2)$ & $26.3(23.2)$ & $-36.2(29.3)^{b, c}$ \\
\hline$P$ value $^{\mathrm{d}}$ & .94 & .27 & .30 \\
\hline \multicolumn{4}{|l|}{ FACE-Q Satisfaction With Nose } \\
\hline Functional & $59.8(20.9)$ & $77.5(22.7)$ & $17.9(23.4)^{\mathrm{b}, \mathrm{c}}$ \\
\hline Dual functional + cosmetic & $34.9(15.0)$ & $68.5(19.9)$ & $34.7(27.3)^{\mathrm{b}, \mathrm{c}}$ \\
\hline$P$ value $^{\mathrm{d}}$ & $<.001$ & .04 & $<.001$ \\
\hline \multicolumn{4}{|l|}{ FACE-Q Satisfaction With Nostrils } \\
\hline Functional & $63.2(27.5)$ & $84.3(22.6)$ & $21.4(27.2)^{\mathrm{b}, \mathrm{c}}$ \\
\hline Dual functional + cosmetic & $45.6(25.5)$ & $81.3(22.6)$ & $36.7(26.7)^{\mathrm{b}, \mathrm{c}}$ \\
\hline$P$ value $^{\mathrm{d}}$ & .001 & .52 & .005 \\
\hline \multicolumn{4}{|l|}{ FACE-Q Social Functioning } \\
\hline Functional & $74.4(21.6)$ & $81.4(22.3)$ & $7.04(19.7)^{b}$ \\
\hline Dual functional + cosmetic & $70.9(22.4)$ & $82.5(20.5)$ & $11.6(22.3)^{\mathrm{b}, \mathrm{c}}$ \\
\hline$P$ value $^{\mathrm{d}}$ & .41 & .81 & .25 \\
\hline
\end{tabular}

Abbreviations: MCID, mean clinically important difference; NOSE, Nasal Obstruction Symptom Evaluation.

${ }^{\text {a }}$ NOSE and FACE-Q scores at baseline and time of last follow-up for those receiving purely functional septorhinoplasty $(n=120)$ vs dual functional and cosmetic septorhinoplasty $(n=34)$.

bStatistically significant change within each cohort.

c Clinically significant change was determined by a change exceeding the MCID for each scale: NOSE score MCID, 30; FACE-Q Satisfaction With Nose MCID, 11.1; Satisfaction with Nostrils MCID, 14.0; and Social Functioning MCID, 10.9.

${ }^{d}$ The $P$ value rows indicate the statistical difference between the cohorts.

\section{Figure 3. Preoperative and Postoperative Results for Patients With Follow-up at 1 Year}

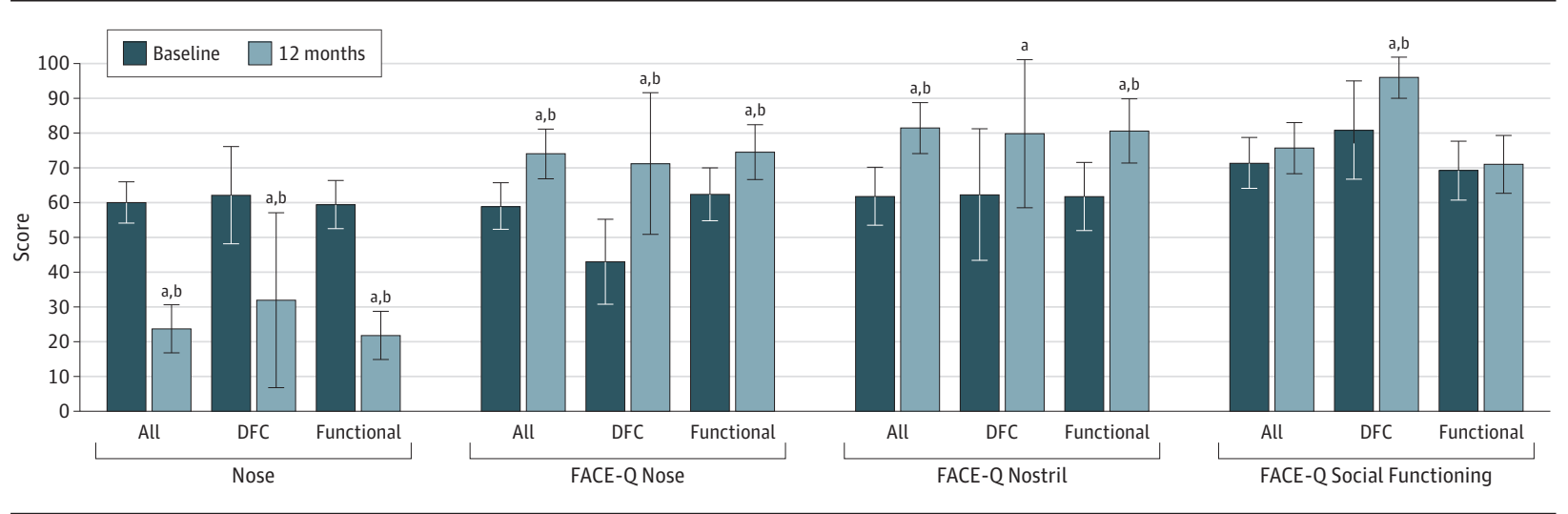

The mean $(95 \% \mathrm{Cl})$ preoperative and 12 -month postoperative Nasal Obstruction Symptom Evaluation (NOSE) and FACE-Q scores are shown for all patients combined (all; $n=42$ ), dual functional and cosmetic septorhinoplasty patients (DFC; $n=8)$, and purely functional septorhinoplasty patients $(n=34)$.
${ }^{a}$ Clinically significant change from baseline. The definitions of clinically significant change in each category are detailed in Results.

b Statistically significant change. improvements in this measure following surgery. Despite this, both groups achieved statistically and clinically significant improvements in FACE-Q Satisfaction With Nose scores, and the purely functional group continued to have significantly higher scores at follow-up (Table 2). Similarly, the DFC group had significantly lower baseline FACE-Q Satisfaction With Nostrils scores and greater score improvements (Table 2). At follow-up there was no significant score difference between the groups, and both cohorts demonstrated clinically and statistically significant score improvements (Table 2). There were no significant differences in baseline or postoperative FACE-Q Social Functioning scores, and both groups demonstrated statistically significant improvements (Table 2). Interestingly, only the DFC group had a clinically significant improvement in FACE-Q Social Functioning score (Table 2). At 12-month follow-up, the DFC group demonstrated both a statistically and clinically significant improvement in FACE-Q Social Functioning score (Table 2), while the combined DFC and FSRP group and FSRP only group had neither a statistically or clinically significant change (Figure 3).

Forty-two patients had both preoperative and 12-month postoperative data. Of these patients, 34 underwent FSRP, and 8 underwent DFC. Figure 3 demonstrates the mean NOSE and FACE-Q scores (Satisfaction With Nose, Satisfaction With Nostrils and Social Functioning) for all patients with follow-up at 1 year as well as results by rhinoplasty type. All groups demonstrated both statistically and clinically significant improvements in NOSE and FACE-Q Satisfaction With Nose scores (Figure 3). FACE-Q Satisfaction With Nostrils changes were all clinically significant, although the DFC group did not reach statistical significance, likely owing to the small number of patients in this group (Figure 3). 


\section{Discussion}

While the emphasis of FSRP is on improving NAO, the surgeon must also consider aesthetic consequences of surgical interventions. As increasing importance is placed on accurately and consistently measuring surgical outcomes, validated disease-specific and global health-related quality-of-life measures for NAO have been established, while evaluation tools for cosmetic outcomes have lagged., ${ }^{1,3,12}$ The patient's perception of their nasal appearance following surgery is a critical aspect of the surgical outcome that must also be evaluated. While prior attempts to quantify a patient's perception of their nasal appearance following FSRP have been made using various rating scales and questionnaires, these measures were not validated or standardized and have not been shown to be reliable or capable of detecting clinical change. ${ }^{7,13}$ The newly developed FACE-Q scales offer an important adjunct to surgical outcomes testing. The scales were developed using both patient and expert input. They have undergone rigorous psychometric testing, and have been found to be valid, reliable, and capable of detecting change. ${ }^{5}$ With the advent of this tool, the aesthetic impact of FSRP for NVD can more accurately be studied.

Nasal valve dysfunction is a commonly overlooked cause of NAO, which, if not identified and properly treated, can lead to surgical failure. ${ }^{2}$ Spreader grafts have previously been found to be associated with improved NAO that is caused by narrowing of the internal nasal valve, and this finding is again demonstrated in the present study by the significant improvement in NOSE scores following FSRP with spreader graft placement. ${ }^{3,4}$ Despite the success in treating internal nasal valve narrowing, spreader graft use has been limited by the concern of widening the nasal dorsum and creating an undesirable cosmetic result. Ingels et $\mathrm{al}^{14}$ found that the nasal dorsum was in fact widened by $6 \%$ following spreader graft placement, although no patients noted the widening when given the opportunity to do so. ${ }^{14}$ While the nasal dorsum may be slightly wider, it is unclear whether this widening translates into an aesthetic deficit in the patient-perceived nasal appearance, and thus a standardized and validated patient-reported outcome measure is necessary to fully understand this.

The present study demonstrates that patient perception of nasal appearance improves following surgery with spreader graft placement, even in purely functional cases. While the impact of nasal width is not specifically investigated herein, we found that overall satisfaction with nasal appearance significantly improved postoperatively compared with baseline. FACE-Q Satisfaction with Nose and Satisfaction With Nostrils scores were significantly improved from baseline at time of last follow-up and at the 2-, 4-, 6- and 12-month follow-up time points, with stability in follow-up scores over time. We saw a drop-off in the number of patient responses over time, and this was attributed to the fact that many patients had not yet reached their next follow-up time point when this study was performed. However, 42 patients did complete 12-month surveys, and 57 patients completed 6-month surveys. While longterm data are important to demonstrate the durability of the results over time, it is interesting to note that in this study, improvements in the FACE-Q scores were seen at 2 months and that this result remained stable through the 12-month follow-up period.

As might be expected, patients undergoing DFC started with lower FACE-Q Satisfaction With Nose and Satisfaction With Nostrils scores than the purely functional group (FSRP), likely driving their request for cosmetic changes. The DFC group experienced greater improvements in patientperceived nasal aesthetics than the FSRP group, though both groups achieved clinically and statistically significant improvements in these scores (Table 2). There was no significant difference in postoperative FACE-Q Satisfaction with Nostrils scores between the groups; however, the lower FACE-Q Satisfaction with Nose scores in the DFC group persisted, perhaps owing to different expectations or greater sensitivity to imperfections.

When looking at all patients together, FACE-Q Social Functioning scores demonstrated a statistically significant improvement but did not reach clinical significance (Table 1 and Figure 3). However, when patients were divided into those receiving FSRP alone and those receiving DFC, the DFC group demonstrated a clinically significant improvement (Table 2 and Figure 3). The modest, not clinically significant, change in the FSRP group may be owing to the fact that this patient population's presenting complaint was NAO, rather than nasal appearance, and thus their nasal appearance likely had little impact on their social functioning. On the other hand, the DFC group was bothered by both NAO and nasal appearance, and thus surgery may have had a greater impact on their social functioning. This suggests that patient perception of nasal appearance may affect social functioning in some patients and that surgeons should appreciate the possible association between nasal appearance and social functioning to ensure that procedures targeted to improve breathing do not have a negative impact on patient-perceived nasal appearance.

In this study, the group with spreader grafts alone and the group with spreader grafts plus other graft types both had significant postoperative improvements in NOSE and FACE-Q scores, without any significant differences between the 2 cohorts. This suggests that the improvement in FACE-Q scores were not due to the placement of other graft types. While further research is needed to determine the impact of each individual technique on both functional and aesthetic outcomes, in isolation, spreader grafts were associated with improved symptoms of NAO without negatively affecting patients' perceptions of their nasal appearance.

\section{Limitations}

While this study demonstrates that both patient-perceived nasal function and aesthetics improve following FSRP with spreader graft placement, this study has limitations. First, a major concern that both patients and surgeons have with spreader graft placement is widening of the nasal dorsum. This study does not directly measure the width of the nasal dorsum before or after surgery and does not specifically assess the patient's satisfaction with their nasal dorsum in isolation. Instead, this study shows that spreader grafts can be used to improve nasal breathing without negatively affecting the 
patient's overall perception of their nasal aesthetics. Further studies are needed to isolate the impact of each grafting technique on both functional and aesthetic outcomes.

Another limitation is the decreased number of patients with follow-up at the longer postoperative time points. This is attributed primarily to the fact that many patients had not reached the next follow-up time point when the present study was performed. However, we surmise that the results would be unchanged, given the stability of follow-up results over time. Finally, another limitation is the inherent bias that comes with self-reported subjective measures in which responses can be biased by a desire to please the surgeon, patient mood, or other subconscious factors.

\section{Conclusions}

Despite concerns that placement of spreader grafts for the treatment of NAO due to nasal valve compromise during FSRP may have a negative impact on nasal aesthetics, this study shows that both symptoms of NAO and patient satisfaction with their nasal appearance significantly improved after surgery. This study provides a quantitative assessment of nasal appearance and nasal function, demonstrating that FSRP with spreader graft placement for the treatment of internal nasal valve narrowing is associated with improved NAO without negatively affecting patient-perceived nasal aesthetics.

\section{ARTICLE INFORMATION}

Accepted for Publication: November 24, 2018

Published Online: March 21, 2019.

doi:10.1001/jamafacial.2018.2118

Author Contributions: Dr Fuller had full access to all of the data in the study and takes responsibility for the integrity of the data and the accuracy of the data analysis.

Study concept and design: Fuller, Lindsay. Acquisition, analysis, or interpretation of data: All authors.

Drafting of the manuscript: Fuller.

Critical revision of the manuscript for important intellectual content: All authors.

Statistical analysis: Fuller.

Administrative, technical, or material support:

Levesque, Lindsay.

Study supervision: Lindsay.

Conflict of Interest Disclosures: None reported.

\section{REFERENCES}

1. Rhee JS, Poetker DM, Smith TL, Bustillo A, Burzynski M, Davis RE. Nasal valve surgery improves disease-specific quality of life. Laryngoscope. 2005;115(3):437-440. doi:10.1097/01. mlg.0000157831.46250.ad

2. Rhee JS, Weaver EM, Park SS, et al. Clinical consensus statement: diagnosis and management of nasal valve compromise. Otolaryngol Head Neck
Surg. 2010;143(1):48-59. doi:10.1016/j.otohns.2010. 04.019

3. Fuller JC, Levesque PA, Lindsay RW. Assessment of the EuroQol 5-dimension questionnaire for detection of clinically significant global health-related quality-of-life improvement following functional septorhinoplasty. JAMA Facial Plast Surg. 2017;19(2):95-100. doi:10.1001/ jamafacial.2016.1410

4. Toriumi DM. Middle nasal vault anatomy and clinical review of spreader grafts. Presented at the Annual Fall Meeting of the American Academy of Facial Plastic and Reconstructive Surgery; September 14-16, 1995; New Orleans, LA.

5. Klassen AF, Cano SJ, East CA, et al. Development and psychometric evaluation of the FACE-Q scales for patients undergoing rhinoplasty. JAMA Facial Plast Surg. 2016;18(1):27-35. doi:10.1001/jamafacial. 2015.1445

6. Vanderbilt University. Research Electronic Data Capture website. https://projectredcap.org/. Accessed February 16, 2019.

7. Lindsay RW. Disease-specific quality of life outcomes in functional rhinoplasty. Laryngoscope. 2012;122(7):1480-1488. doi:10.1002/lary.23345

8. Stewart MG, Witsell DL, Smith TL, Weaver EM, Yueh B, Hannley MT. Development and validation of the Nasal Obstruction Symptom Evaluation (NOSE) scale. Otolaryngol Head Neck Surg. 2004;130(2): 157-163. doi:10.1016/j.otohns.2003.09.016
9. Rhee JS, Sullivan CD, Frank DO, Kimbell JS, Garcia GJ. A systematic review of patient-reported nasal obstruction scores: defining normative and symptomatic ranges in surgical patients. JAMA Facial Plast Surg. 2014;16(3):219-225. doi:10.1001/ jamafacial.2013.2473

10. Timperley D, Srubisky A, Stow N, Marcells GN, Harvey RJ. Minimal clinically important differences in nasal peak inspiratory flow. Rhinology. 2011;49 (1):37-40.

11. Norman GR, Sloan JA, Wyrwich KW. Interpretation of changes in health-related quality of life: the remarkable universality of half a standard deviation. Med Care. 2003;41(5):582-592. doi:10 1097/01.MLR.0000062554.74615.4C

12. Rhee JS, Arganbright JM, McMullin BT, Hannley $M$. Evidence supporting functional rhinoplasty or nasal valve repair: a 25-year systematic review. Otolaryngol Head Neck Surg. 2008;139(1):10-20. doi:10.1016/j.otohns.2008.02.007

13. Toriumi DM, Josen J, Weinberger M, Tardy ME Jr. Use of alar batten grafts for correction of nasal valve collapse. Arch Otolaryngol Head Neck Surg. 1997;123(8):802-808. doi:10.1001/archotol.1997. 01900080034002

14. Ingels KJ, Orhan KS, van Heerbeek N. The effect of spreader grafts on nasal dorsal width in patients with nasal valve insufficiency. Arch Facial Plast Surg. 2008;10(5):354-356. doi:10.1001/archfaci.10.5.354 